\title{
Tiresias: A low-cost networked UWB radar system for in-home monitoring of dementia patients
}

\author{
Alan Bannon \\ EEE. Imperial College London \\ London, UK \\ a.bannon@imperial.ac.uk
}

\author{
Adrien Rapeaux \\ EEE. Imperial College London \\ London, UK \\ adrien.rapeaux13@imperial.ac.uk
}

\author{
Timothy Constandinou \\ EEE. Imperial College London \\ London, UK \\ t.constandinou@imperial.ac.uk
}

\begin{abstract}
This paper describes Tiresias, a low-cost, unobtrusive networked radar system designed to monitor vulnerable patients in domestic environments and provide high quality behavioural and health data. Dementia is a disease that affects millions worldwide and progressively degrades an individual's ability to care for themselves. Eventually most people living with dementia will need to reside in assisted living facilities as they become unable to care for themselves. Understanding the effects dementia has on ability to self-care and extending the length of time people living with dementia can remain living independently are key goals of dementia research and care. The networked radar system proposed in this paper is designed to provide high quality behavioural and health data from domestic environments. This is achieved using multiple radar sensors networked together with their data outputs integrated and processed to produce high confidence measures of position and movement. It is hoped the data produced by this system will both provide insights into how dementia progresses, and also help monitor vulnerable individuals in their own homes, allowing them to remain independent longer than would otherwise be possible.

Index Terms-Radar, UWB, indoor tracking, networked sensing, long-term monitoring, dementia, integrated care.
\end{abstract}

\section{INTRODUCTION}

Dementia progressively reduces the ability of those affected by it to live independently. Affecting around 50 million people worldwide [1], the effects of dementia place a heavy toll on healthcare systems and remains a leading cause of patients requiring residence in assisted living facilities. Longterm monitoring of the behaviour and well-being of patients with dementia can allow clinicians to assess both disease progression, and the effects of any interventions or changes in circumstance.

High quality behavioural data can be generated by monitoring patients with video cameras using computer vision or manual behavioural labelling. However, this approach is extremely obtrusive, comes with many privacy concerns, and would be very unlikely to be acceptable to many people living with dementia [2], [3]. On the other end of the obtrusiveness scale are sensor systems for individual actions, such as use of appliances, opening of doors and windows, or presence in a [4]. Unfortunately, with the unobtrusiveness of these systems comes a reduction in the quality of the data gathered. Point events such as these can give an insight into behaviour and

Work supported by the UK Dementia Research Institute. independence, but often require very dense sampling of the space with many devices to be effective [5].

Radar offers the strengths of both approaches by supplying high quality location and movement data, while being unobtrusive and maintaining as much privacy as possible within the scope of the desired monitoring. Additionally, radar can provide biometric data such as respiration and heart rate [6] that allow for ever greater insight into the health of patients. This paper describes Tiresias, a low-cost, unobtrusive networked radar system designed to monitor vulnerable patients in domestic environments and provide high quality behavioural and health data.

Central to the design of the proposed system is the $\mathrm{X} 4$ radar module produced by the NOVELDA AS company [7]. The X4 radar is an ultra-wideband (UWB) pulsed radar with operating modes in the 6-8.6 and 7.25-10.2 GHz ranges to allow for worldwide regulatory compliance [8]. Available as a commercial off the shelf (COTS) product, the X4 carries a CE mark and is fully FCC compliant. A single radar sensor has a field of view limited only by the geometry of its surrounding space. In a domestic environment it is usually not possible to observe every area of interest from a single point, and thus, for complete coverage multiple sensors must be used. With multiple sensors also comes the opportunity to combine overlapping coverage to both increase data quality, and provide measures such as $2 \mathrm{D}$ positioning not available with a single axis radar sensor.

The remainder of this paper is organised as follows: Section II describes the design and implementation of the system and data processing; Section III covers the data collection and testing performed so far; Section IV presents and discusses the results obtained; Section V places the system in the wider scope of ongoing work; Section VI concludes and summarizes the work.

\section{Methods}

The X4 UWB radar sensor is a single transceiver sensor, meaning the output data is along a single range dimension. While placing objects in range is straightforward, in order to place an object in 2 dimensions, for example an individual in a room, two sensors must be used with overlapping fields of view. By networking sensors together via a local WiFi we can allow sensor nodes to be placed freely and maximise coverage. 


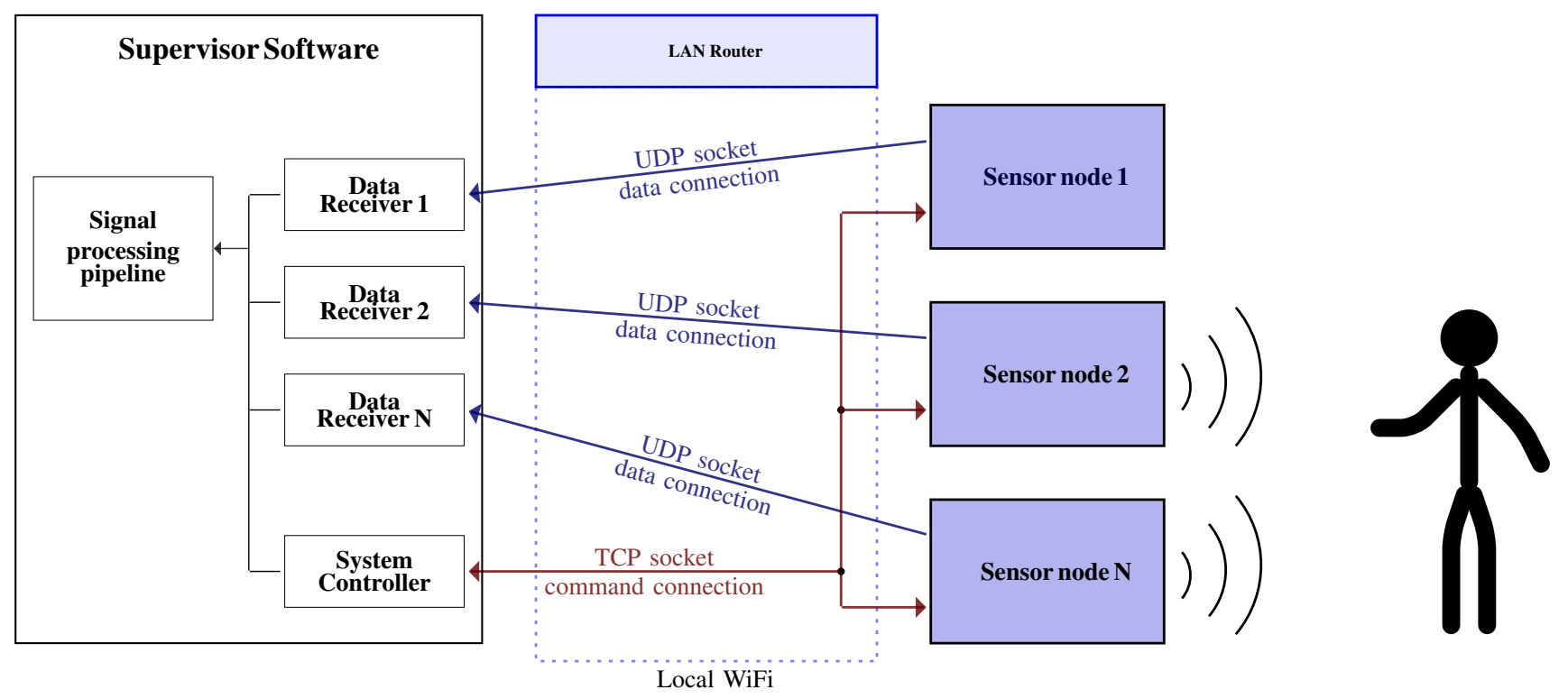

Fig. 1. High level system diagram showing networking connections and data flow.

While sensing is dispersed, a central supervisor is used to collate and process the data gathered from the networked nodes. To maintain synchronicity and ensure that data does not become skewed due to timing errors between the elements of the system, timestamps are used at data generation and the collected data aligned to remove any skew.

The received and aligned data requires a multi-stage processing pipeline to yield useful results. The raw data contains reflections from every object in the field of view of the sensor, including static objects such as furniture and walls, and these must be differentiated from moving objects of interest, such as people, in order to locate and detect them.

\section{A. Radar system and networking}

As shown in Fig. 2, the hardware of each sensor node is comprised of an $\mathrm{X} 4$ radar sensor, several interface and routing PCBs providing connections, indicators, and user interface buttons, and a Raspberry Pi Zero W. Due to using COTS components almost exclusively the total hardware cost of each sensor node is around £80. The X4 communicates over SPI with the Raspberry Pi, allowing the Pi to configure and control the functionality of the $\mathrm{X} 4$, as well as receive radar data frames. The Raspberry Pi runs Tiresias sensor node software continuously from startup. The node software is mainly focused on networking, and apart from collecting the raw radio-frequency (RF) data from the radar does not do any data processing. Rather, from startup the node software scans the local network and attempts to find a device that responds correctly to a handshake request over a TCP socket. The supervisor software, which can run on any WiFi device that supports Python, will confirm the handshake upon receiving the request on a specific port and start issuing commands to the sensor node. In turn, the sensor node will follow commands from the supervisor software on how to configure the $\mathrm{X} 4$,

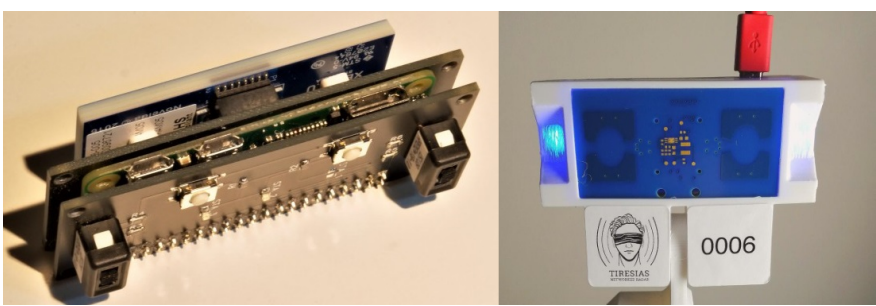

Fig. 2. (left) Internal electronics stack, from top: $\mathrm{X} 4$ radar, radar interface board, Raspberry Pi Zero W, user interface board. (right) Tiresias sensor node 0006 on adjustable 3D printed mount. Note the two differential X4 patch antennas visible against the blue substrate of the sensor.

when to start transmitting data, and to which port to send the data. Data is sent over UDP sockets to decrease network overhead and allow for higher data rates. When a sensor node receives a transmit command, it will also receive a unique port number to send the data toward. The supervisor software will start a separate data handling process to receive incoming data frames, and attach it to the appropriate port. This data flow is described in Fig. 1.

\section{B. Data alignment}

With elements separated both physically and in independent processes, it is necessary to ensure that the data collected from the networked sensor nodes is correctly temporally aligned. To achieve this the system operates within discrete $100 \mathrm{~ms}$ time epochs. To allow for correction, each sensor node attaches a timestamp to every data frame as soon as it is collected from the $\mathrm{X} 4$ radar. The timestamps have $\sim 1 \mathrm{~ms}$ precision and are all referenced to the UNIX time epoch [9] using the system clock set over the network using NTP. On receipt of a data frame from a node the supervisor software attempts to place the frame in the correct $100 \mathrm{~ms}$ time epoch. Each time epoch is 


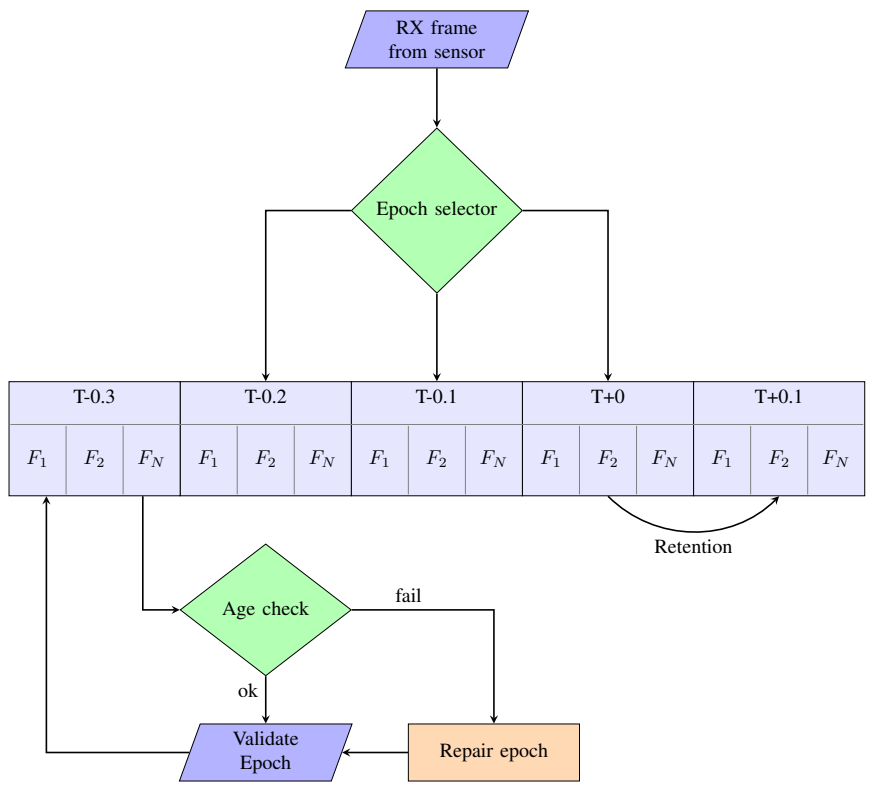

Fig. 3. Data alignment process flowchart. In this instance $M=3$ and $H=3$.

implemented as a data object containing $N$ data frames, their associated collection timestamps, and information on how old each frame is relative to the epoch's start. The exact number of epochs retained in memory is configurable, with the current "working" epoch denoted $T+0$ and previous epochs $T-(M *$ 0.1 ) for $M$ epochs. The future epoch, $T+0.1$ is created during the current epoch and is initialised with all the data from the current epoch. If a change is made to the current epoch before it ends the change is also propagated to the future epoch.

As new frames are received their timestamp is compared against the current and previous epochs. If the timestamp falls into an epoch that has a frame older than the received frame the older frame is replaced. If the replaced frame had previously been carried forward during a future epoch creation, a check is made to see if the new frame should also be propagated forward. If an even newer frame has been received upstream of the frame in question, propagation will proceed up to the newest frame.

Finally, at a configurable point $H$ epochs behind the current epoch, a check is performed on each epoch. If all the frames were updated during the epoch, the epoch is validated and passed to the next processing stage. However, if one or more frames were not updated during the epoch a repair will be attempted on the stale frame(s). For the repair, neighboring epochs are searched to determine the closest valid frame (as this may be after the epoch in question). If the closest frame is sufficiently close, only a few ms into the next epoch, it will be back-propagated into the frame in question. If not, the two closest valid frames will be averaged. While averaging provides data that may be recoverable during the following processing, it does potentially reduce the SNR of any moving reflectors in the data. This process is shown graphically in Fig. 3.

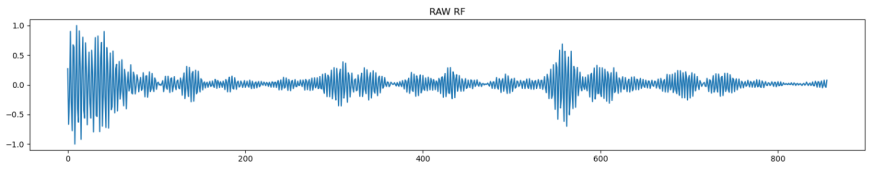

Fig. 4. Raw RF data captured during a test in a domestic environment

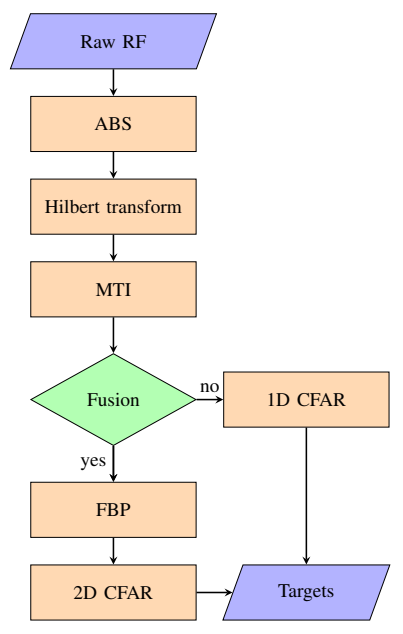

Fig. 5. Signal processing pipeline flowchart

\section{Signal processing and data fusion}

The X4 radar directly outputs digitised RF measurements, an example of which is shown in Fig. 4, To find reflections from targets of interest - in this case individuals - a signal processing pipeline is implemented as follows: first, the absolute value of the sinusoidal RF signal is taken; a power envelope is then generated using a Hilbert Transform; novel reflections are then extracted using a three pulse canceller moving target indicator filter (TPC-MTI); if there are overlapping fields of view from other sensors these are combined into a single representation of the scene using filtered back projection [10]; finally a $1 \mathrm{D}$ or $2 \mathrm{D}$ constant false alarm rate (CFAR) detector is used to suppress spurious detections in the scene. This process is shown graphically in Fig. 5.

\section{EXPERIMENTAL SETUP}

Tests were performed with the system in both laboratory and domestic environments. In the laboratory environment measurements were taken to validate the accuracy and validity of positional detection by having a subject walk to a series of points marked at $1 \mathrm{~m}$ intervals.

To validate the system in a domestic environment, the system was installed in the apartment of one of the authors ${ }^{1}$. In this way the outputs of the system were validated, and in large part developed, in a completely realistic domestic environment. Movement and localization where measured during several trials involving walking between marked locations and unscripted normal domestic activities. The dimensions of the

\footnotetext{
${ }^{1}$ Please note that parts of this work were undertaken during a national lockdown due to Covid-19 pandemic, and as such lab facilities where not always accessible.
} 


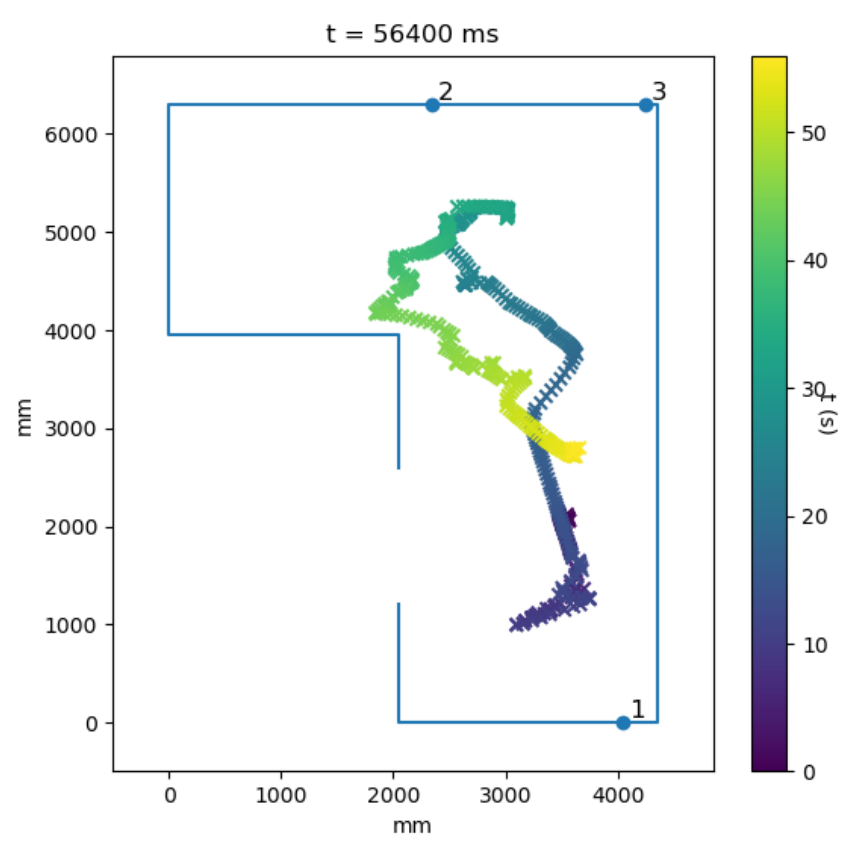

Fig. 6. 2D-CFAR results showing an individual's walking history in a domestic environment. Sensor locations $1,2,3$ are also shown.

space and positions of the sensors are detailed, along with a track depicting an individual's path when walking through the room, in Fig. 6.

\section{RESUlTS AND DISCUSSION}

Verification and calibration of the system in a laboratory environment proceeded without issues. Fig. 7 shows the CFAR output of the system as an individual walks to a series of marks at $1 \mathrm{~m}$ intervals. While there is some detection noise downrange of the subject it is inconsistent and can be rejected in the track formation process. Several such datasets were recorded with a range of radar sensor mounting angles to assess how inclination to the vertical affected the quality of the data produced. It was found that a simple 45 degree inclination to the vertical produced the most consistent results over the $5 \mathrm{~m}$ test range.

During the deployment in the domestic environment several datasets were collected of both scripted movements to premeasured positions and also unscripted domestic activities. Fig. 6 shows one such period of unscripted domestic activity. $\sim 56 \mathrm{~s}$ of location history is shown, denoted by colour, as the individual pauses, and walks between locations. Note that although sections of the track overlap, during some sections there appears to be some noisy lateral movements. This noise is most likely due to the cyclic nature of human gait causing the reflection from a walking individual to change over time, and cause the detected position to oscillate. Despite this, an accurate track is maintained, and presence at and movement between various locations - cooker, sink, chair- can be extracted from the data.

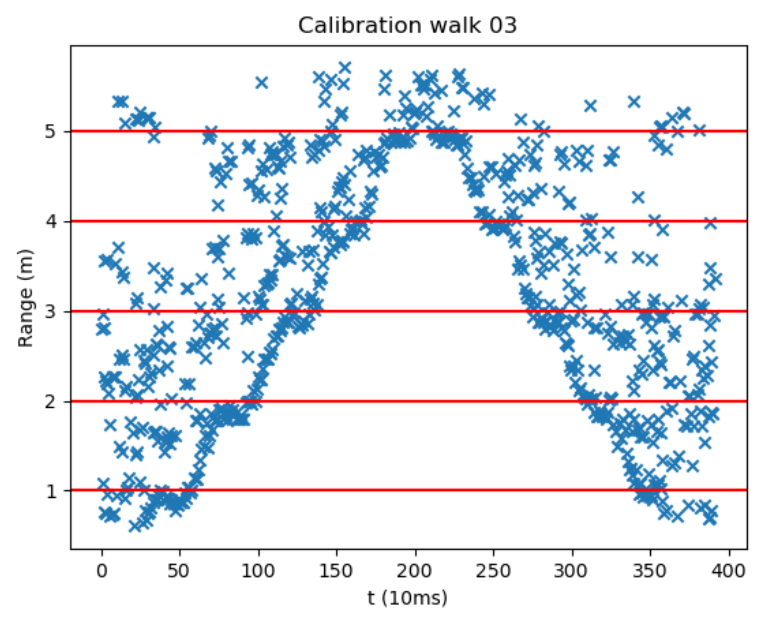

Fig. 7. Calibration data showing 1D-CFAR output as an individual walks away and toward a sensor, pausing at $1 \mathrm{~m}$ intervals.

\section{FUTURE WORK}

Gathering more data would be extremely valuable to further validate the system in domestic environments. Data collection is currently ongoing in the researchers' own homes with healthy volunteers, as this data is useful to explore the strengths and limitations of the system before progressing to more challenging targets such as elderly people.

Moving forward there are additional metrics that can be extracted from the data collected by Tiresias. Biometric monitoring has been shown to work with X4 data [6], and would provide an additional set of metrics to both aid locating stationary people in the observable scene provide more data on the health of patients. Moreover, biometric markers could help identify and distinguish different individuals being monitored in the same space without relying on wearable sensors or video.

Changes to walking gait are also known to be associated with the progression of dementia [11], [12], and as gait patterns are specific to individuals would also be useful to help identify and distinguish between different individuals.

Finally, the object tracking used to maintain a consistent track is currently functional but basic. A more complex adaptive tracking process is being developed with the intention of forming the basis for a future publication.

\section{CONCLUSION}

This paper has detailed the design, operation, and performance of a low-cost networked UWB radar system for monitoring vulnerable individuals in domestic environments. The physical hardware has been considered, and the data flow has been described from the sensor, through to networking and detection. The proposed system is low cost and has low computational requirements while maintaining worldwide regulatory compliance. The data produced has been shown to be of sufficient quality to provide insights into the behaviour of individuals in a domestic environment. It is hoped that the 
system will continue into further validation and deployments to make a positive impact on the lives of those living with dementia.

\section{REFERENCES}

[1] World Health Organization and others, "Towards a dementia plan: a WHO guide," 2018.

[2] A. Braeken, P. Porambage, A. Gurtov, and M. Ylianttila, "Secure and efficient reactive video surveillance for patient monitoring," Sensors (Basel, Switzerland), vol. 16, no. 1, p. 32, Jan 2016, 26729130[pmid]. [Online]. Available: https://pubmed.ncbi.nlm.nih.gov/26729130

[3] O. Gelonch, M. Ribera, N. Codern-Bové, S. Ramos, M. Quintana, G. Chico, N. Cerulla, P. Lafarga, P. Radeva, and M. Garolera, "Acceptability of a lifelogging wearable camera in older adults with mild cognitive impairment: a mixed-method study," $B M C$ Geriatrics, vol. 19, no. 1, p. 110, Apr. 2019. [Online]. Available: https://doi.org/10.1186/s12877-019-1132-0

[4] H. Rostill, R. Nilforooshan, A. Morgan, P. Barnaghi, E. Ream, and T. Chrysanthaki, "Technology integrated health management for dementia," British Journal of Community Nursing, vol. 23, no. 10, pp. 502-508, 2018, pMID: 30290728. [Online]. Available: https://doi.org/10.12968/bjen.2018.23.10.502

[5] D. J. Cook, A. S. Crandall, B. L. Thomas, and N. C. Krishnan, "CASAS: A Smart Home in a Box," Computer, vol. 46, no. 7, Jul. 2013. [Online]. Available: https://www.ncbi.nlm.nih.gov/pmc/articles/PMC3886862/

[6] Z. Chen, A. Bannon, A. Rapeaux, and T. G. Constandinou, "Towards robust, unobtrusive sensing of respiration using ultra-wideband impulse radar for the care of people living with dementia," bioRxiv, 2020. [Online]. Available: https://www.biorxiv.org/content/early/2020/12/15/ 2020.12.14.422564

[7] "The human presence sensor," Jan 2021. [Online]. Available: https://novelda.com/

[8] N. Andersen, K. Granhaug, J. A. Michaelsen, S. Bagga, H. A. Hjortland, M. R. Knutsen, T. S. Lande, and D. T. Wisland, "A 118-mw pulse-based radar soc in 55-nm cmos for non-contact human vital signs detection," IEEE Journal of Solid-State Circuits, vol. 52, no. 12, pp. 3421-3433, 2017.

[9] D. M. Ritchie, "The unix system: The evolution of the unix time-sharing system," AT T Bell Laboratories Technical Journal, vol. 63, no. 8, pp. 1577-1593, 1984.

[10] R. M. Mersereau and A. V. Oppenheim, "Digital reconstruction of multidimensional signals from their projections," Proceedings of the IEEE, vol. 62, no. 10, pp. 1319-1338, 1974.

[11] O. Beauchet, G. Allali, G. Berrut, C. Hommet, V. Dubost, and F. Assal, "Gait analysis in demented subjects: Interests and perspectives," Neuropsychiatric disease and treatment, vol. 4, no. 1, pp. 155-160, Feb 2008, 18728766[pmid]. [Online]. Available: https: //pubmed.ncbi.nlm.nih.gov/18728766

[12] R. A. Hackett, H. Davies-Kershaw, D. Cadar, M. Orrell, and A. Steptoe, "Walking Speed, Cognitive Function, and Dementia Risk in the English Longitudinal Study of Ageing," Journal of the American Geriatrics Society, vol. 66, no. 9, pp. 1670-1675, 2018, _eprint: https://agsjournals.onlinelibrary.wiley.com/doi/pdf/10.1111/jgs.15312. [Online]. Available: https://agsjournals.onlinelibrary.wiley.com/doi/abs/ $10.1111 /$ jgs. 15312 